\title{
Capacidad vital, talla, peso y superficie corporal en dos poblaciones adultas sanas, residentes de altura del Norte Grande de Chile: 98 nativos del bofedal de Isluga (altura promedio $3800 \mathrm{~m}$ ) y 351 residentes del campamento cuprífero de Chuquicamata (2800 m)
}

Raimundo Santolaya ${ }^{1}$, José Araya ${ }^{2}$ y Raúl Prieto ${ }^{3}$

\section{Introducción}

El hombre que habitaba nuestro continente a la llegada de los españoles es el producto, casi con certeza, de sucesivas inmigraciones de paleomongoloides que desde el noreste de Asia cruzaron por el actual estrecho de Bering, durante la cuarta y la última glaciación, conocida como de Wisconsin, correspondiente a la europea de Würm (Panyella 1975; Mostny 1976). Estas oleadas de cazadores y recolectores se iniciaron probablemente hace más de 40000 años y finalizaron hace unos 10000 , cuando el puente terrestre que unía Siberia, en el noreste de Asia con Alaska en el norte de América, desapareció bajo las aguas que subieron su nivel en 60 a 100 m, debido a la fusión del hielo, al iniciarse el actual interglaciar (D'Orbigny 1944; Hopkins 1962; Kauffmann-Doig 1978).

Se trataba de un tipo de hombre aclimatado a una planicie alta, seca y fría: la Mongolia, de $1500 \mathrm{~m}$ de altura. Era un hombre de estatura mediana a pequeña; con considerable desarrollo del tórax que configuraba una arquitectura ovalar, clásicamente señalada como óptima para la aclimatación al frío (Coon et al. 1950; Monge y Monge 1967).

Sin embargo, las características somáticas de los hombres de las diversas oleadas poseían variables que hasta hoy pueden descubrirse en sus descendientes distribuidos desde Alaska hasta Patagonia. Al parecer, las primeras oleadas fueron más dolicocéfalas y de estaturas mayores, haciéndose más braquicéfalas las que siguieron y de menor talla. La última, de la que parecen derivar los esquimales, son de muy baja estatura y predominancia dolicocefálica. Los pueblos llamados neoamerindios, habitantes sudpacíficos desde México hasta los Andes meridionales,

1 Centro de Investigaciones Ecobiológicas y Médicas de Altura, Hospital Roy H. Glover, Chuquicamata, CHILE.

2 Servicio Anestesiología, Hospital Roy H. Glover. Miembro del Centro de Investigaciones, Chuquicamata, CHILE.

3 Hospital Roy H. Glover, Chuquicamata, CHILE. incluido el pueblo araucano, corresponderían a las migraciones intermedias y sus caracteres mongoloides: Talla mediana y braquicefalia predominante, pueden verificarse hasta hoy (Panyella 1975).

El más antiguo vestigio humano americano, con cronología verificada, es hasta hoy el indio de Norteamérica cuyos 37000 años AP fueron descritos por Hopkins (1962). Entre los pueblos andinos McNeisch (cit. en Kauffmann-Doig 1978) pudo comprobar en 1968 que en esta zona de Ayacucho, en los Andes Centrales, existió un hombre preagrícola hace 22000 años. Cardich (1960) demostró con ulterior confirmación con $\mathrm{C}^{14}$ que en Lauricocha habitó un hombre preagrícola a $4000 \mathrm{~m}$ de altura hace $9525 \pm 250$ años AP, por lo menos.

Tanto los paleomongoloides que migraron a América como los que permanecieron en su hábitat originario evolucionaron hasta hoy. Muchos de ellos debieron modificarse adaptativamente a los diversos ecosistemas que encontraron, algunos muy exigentes, como ocurrió en los Andes. Existe amplia evidencia de que el hombre andino posee características estructurales y funcionales particulares que le permiten una adecuada y eficiente sobrevivencia en su medio ambiente. Estos rasgos comprenden al organismo como un todo a los distintos órganos y sistemas y muy especialmente a la intimidad bioquímica y ultraestructural de las células (Hurtado 1964; Santolaya 1985).

A la modificación del fenotipo se habrán agregado otras características del genotipo. No sólo adaptativas sino también producto del mestizaje, progresivo aunque no homogéneo, que caracteriza a los pueblos del continente por las inmigraciones ulteriores de razas caucasoides, negroides y polinésicas.

Es sabido que en las zonas de altura los individuos de todas las razas tienden a un crecimiento lento y prolongado, con retardo de la iniciación del crecimiento rápido de la adolescencia y menor talla definitiva (Frisancho 1978). Junto a ello, el tamaño y volumen torácico es mayor que el de los habitantes de las tierras bajas de igual etnia y mucho mayor 
que el supuesto para la talla del individuo (Frisancho 1970, 1977). Estas características son útiles en el proceso de aclimatación a la presión barométrica baja de su hábitat. Su mantención y perfeccionamiento en los pueblos andinos constituye un parámetro habitualmente considerado adaptativo. Sin embargo, pudiera además explicar la fácil aclimatación que los pueblos paleomongoloides experimentaron al exigente medio ambiente polar andino y a su baja presión de oxígeno atmosférico, como consecuencia de estas características que en algún grado poseían ya al momento de su inmigración americana.

Iguales modificaciones se obtienen experimentalmente en ratas expuestas entre su $23^{\circ}$ y $44^{\circ}$ día de vida, a presiones barométricas bajas (3450 $\mathrm{m}$ de altura) (Burri y Weibel 1971). Incrementan casi todos los parámetros de los volúmenes pulmonares y de las superficies de intercambio. Además se produce depresión del crecimiento (Cheek et al. 1969; Burri y Weibel 1970) y disminución de peso corporal (Timiras et al. 1957).

El presente trabajo pretende aportar nuevos datos sobre talla, peso y superficie corporal en relación a capacidad vital, importante indicador de volúmenes pulmonares. Resume en forma preliminar las investigaciones realizadas en dos comunidades residentes en asentamiento de altura de los Andes del norte de Chile y su comparación entre sí y con tablas internacionales de uso habitual en al práctica clínica y antropométrica (Kory et al. 1961).

\section{Material y métodos}

Los datos que se presentan fueron obtenidos en dos comunidades residentes de altura del Norte Grande de Chile. Las características de ambos grupos son las siguientes:

a) Grupo Isluga. Se trata de nativos indígenas, de origen aymara, residentes en los diversos pueblos y villorrios de la margen del río Isluga, que transcurre en forma de bofedal en el altiplano chileno, hasta su desembocadura en el salar de Coipasa. Los exámenes fueron realizados en nuestro laboratorio ubicado en la hacienda Cusa Llapu (CORFO) a $3650 \mathrm{~m}$ de altura, en Colchane, Provincia de Iquique, Región de Tarapacá, a $19^{\circ} 15^{\prime} \mathrm{S}$ y $68^{\circ} 48^{\prime} \mathrm{O}$, presión barométrica promedio de $484 \mathrm{mmHg}$.

Los individuos estudiados son pastores parcialmente trashumantes que habitan asentamientos cuyas alturas fluctúan entre 3650 y $4500 \mathrm{~m}$. Viven de acuerdo a sus tradiciones ancestrales, alcanzando con frecuencia alturas mayores para recoger leña en los queñuales o apacentar ganado. Hasta el momento de la investigación ninguno de ellos conocía el mar o había descendido a las tierras bajas.

Los datos que se presentan en esta ocasión complementan publicaciones anteriores (Donoso y Santolaya 1971; Santolaya et al. 1977) y se desprenden de un amplio estudio que comprendió a más de 400 individuos de ambos sexos y de ancho rango de edad que fueron sometidos a anamnesia, examen físico completo, reacción de PPD de dos unidades y, en menor número, a mediciones antroprométricas, hematocrito, electrocardiograma y estudio funcional pulmonar (Donoso et al. 1976; Santolaya y Donoso 1976). En 25 de ellos se practicó además medición de capacidad aeróbica en ciclo ergómetro. Las cifras actuales corresponden a 72 hombres y 42 mujeres cuyas edades fluctuaron entre 15 y 60 años. El peso y talla fueron obtenidos mediante una balanza con cartabón y la superficie, calculada de acuerdo a la carta de superficie corporal de Dubois, preparada por Boothby y Sandiford de la Clínica Mayo. En las 42 mujeres y 56 hombres se practicó estudio espirométrico completo usando un respirómetro de 13.5 lt (Collins). Solo se analizarán los valores de capacidad vital. Se eligió en cada caso el máximo valor obtenido entre dos pruebas de capacidad vital $\mathrm{y}$ dos pruebas de volumen espiratorio forzado.

b) Grupo Chuquicamata. Se trata de trabajadores no mineros y sus familiares, residentes permanentes en el campamento cuprífero de Chuquicamata ubicado a $2800 \mathrm{~m}$, en el desierto marginal de altura de la Provincia de El Loa Región de Antofagasta, a $22^{\circ}$ $15^{\prime} \mathrm{S}$ y $68^{\circ} 55^{\prime} \mathrm{O}$, presión barométrica promedio de $548 \mathrm{mmHg}$.

Los individuos representan una comunidad mayoritariamente de la región, étnicamente heterogénea, comparable a la población chilena de la costa del Norte Grande. Su residencia en la altura, en ningún caso fue menor de dos años. Sus hábitos de vida, de alimentación, de consumo de tabaco son los habituales de la región, con marcada tendencia al sedentarismo de preferencia en las mujeres. Sus salidas a la costa varían mucho individualmente pero ocurren en todos ellos, por lo menos una vez al año.

Se presentan en esta ocasión los valores de peso, talla y superficie corporal obtenidos con igual técnica, en 181 hombres y 170 mujeres cuyas edades fluctuaron entre 18 y 75 años. Se relatan además los valores de capacidad vital calculados en un estudio funcional pulmonar completo, antes y después de inhalar 
aerosol brondilatador. El estudio fue practicado en un espirómetro electrónico modelo 370 Wedge de Picker Int. Co., complementado con un pulmo digitizer 280 y 282: medidor de nitrógeno Delta y medidor de volumen ventilar en máximo modelo 171 de Med. Science Electronic. Estos exámenes forman parte del protocolo de una investigación prospectiva en marcha, que comprende numerosos parámetros clínicos, de aclimatación a altura y de laboratorio, cuyos detalles se han analizado en publicaciones parciales anteriores (Santolaya et al. 1981).

\section{Resultados}

Los resultados obtenidos se presentan en siete tablas y cuatro gráficos.

En las Tablas 1 y 2 se anotan los valores promedios y los rangos de peso (k); talla (cm); superficie corporal $\left(\mathrm{m}^{2}\right)$ y capacidad vital (ml BTPS) obtenidos en 56 hombres y 42 mujeres respectivamente, aparentemente sanas, nativas residentes del altiplano chileno cuya altura media fluctúa entre 3800 y 4100 m.snm.

En las Tablas 3 y 4 se presentan los resultados obtenidos para iguales parámetros en 181 hombres y 170 mujeres respectivamente, residentes permanentes, trabajadores no mineros del campamento de Chuquicamata ubicado a 2800 m.snm.

En la Tabla 5 se resumen los valores promedio de peso y superficie corporal para todos los individuos de ambos grupos.

En las Tablas 6 y 7 se anotan respectivamente para los hombres de ambos grupos y para las mujeres de ambos asentamientos los valores promedio de peso, talla, superficie corporal y capacidad vital, expresada en litros por $\mathrm{m}^{2}$ de superficie y como porcentaje del supuesto ideal deducido para su talla y edad, de la tabla de valores espirométricas normales, habitualmente en uso, de Kory y colaboradores (1961).

En todas las tablas se expresan los datos en relación a grupos etarios, para cada caso.

En los Gráficos 1 a 4 se muestran los valores de capacidad vital obtenidos, expresados como porcentaje del supuesto ideal para cada caso, respecto de la tabla de Kory y colaboradores (1961) y distribuidos según edad. Los Gráficos 1 y 2 se refieren a la población de Isluga, hombres y mujeres, respectivamente. Los Gráficos 3 y 4 exponen los resultados de Chuquicamata para ambos sexos, respectivamente.
La línea paralela al eje de las abscisas, que se inician a la altura 100 del eje de las ordenadas, pretende graficar los valores que se encuentran por debajo o por encima del supuesto ideal normal para la edad, sexo y talla, de acuerdo a la mencionada tabla de Kory y colaboradores (1961).

\section{Comentario}

La muestra cuyo estudio preliminar se presenta en esta ocasión permite obtener algunos datos de interés sobre la antropología física en dos comunidades de altura del norte chileno. El grupo más homogéneo desde el punto de vista étnico, de hábitos de vida y de aclimatación a la altitud, corresponde al formado por los nativos de Isluga. En ellos el promedio de talla entre los 15 y 60 años fluctuó en los hombres entre 163 y $166 \mathrm{~cm}$ y entre las mujeres entre $\mathbf{x x x x}$ y $\mathbf{x x x x}^{4} \mathrm{~cm}$. Estos promedios son ligeramente mayores que los publicados por Monge para indígenas de Huancayo. En esa casuística los hombres de 20 a 24 años habitantes de altura entre 2800 y $4000 \mathrm{~m}$ poseen talla promedio de $160 \mathrm{~cm}$ (Monge et al. 1954). La talla es también mayor que la referida por el mismo autor en 1954 para 53 jóvenes indígenas residentes a más de $3600 \mathrm{~m}$ en el altiplano boliviano. Comparada con la talla promedio publicada por Frisancho y Baker (1970), para jóvenes de Nuñoa, residentes a alturas superiores a $4000 \mathrm{~m}$, de $158.8 \mathrm{~cm}$, la talla de nuestros hombres menores de 20 años y entre 21 y 30 años (Tabla 1), de 163 y $165 \mathrm{~cm}$ respectivamente fue también mayor. Los mismos autores refiere talla promedio de $160 \mathrm{~cm}$ para 50 hombres de Nuñoa de 35 años de edad. Ese grupo etario en nuestra serie de Isluga mostró una talla promedio de $165 \mathrm{~cm}$.

La talla promedio de las mujeres de todas las edades del grupo de Isluga fluctuó escasamente alrededor de $150 \mathrm{~cm}$, cifra que se acerca a la de Frisancho y Baker que en 125 mujeres, cuyas edades variaron de 20 a 35 años, obtiene valores promedio entre 148 y $148.6 \mathrm{~cm}$.

Al comparar las tallas de hombres y mujeres de Isluga con las de Chuquicamata se evidencia que los residentes de este campamento poseen estaturas promedio mayores, en ambos sexos y en todos los grupos etarios. Esta diferencia no puede imputarse solo a la menor altitud de residencia sino

\footnotetext{
4 Nota de la Editora (2009): x = Ininteligible en el original.
} 


\begin{tabular}{|c|c|c|c|c|c|c|c|c|c|}
\hline \multicolumn{10}{|c|}{ Hombres } \\
\hline Edad & $\mathbf{N}^{\mathbf{o}}$ & \multicolumn{2}{|c|}{ Peso (k) } & \multicolumn{2}{|c|}{ Talla (cm) } & \multicolumn{2}{|c|}{ Superficie $\left(\mathbf{m}^{2}\right)$} & \multicolumn{2}{|c|}{ Capacidad vital (ml) } \\
\hline \multirow{2}{*}{$15-20$} & \multirow{2}{*}{13} & \multicolumn{2}{|c|}{55.90} & \multicolumn{2}{|c|}{163.00} & \multicolumn{2}{|c|}{1.59} & \multicolumn{2}{|c|}{5490} \\
\hline & & 46 & 64 & 151 & 170 & 1.38 & 1.74 & 4245 & 6380 \\
\hline \multirow{2}{*}{$21-30$} & \multirow{2}{*}{14} & \multicolumn{2}{|c|}{58.60} & \multicolumn{2}{|c|}{165.00} & \multicolumn{2}{|c|}{1.64} & \multicolumn{2}{|c|}{5577} \\
\hline & & 46 & 66 & 154 & 175 & 1.44 & 1.76 & 3896 & 6860 \\
\hline \multirow{2}{*}{$31-40$} & \multirow{2}{*}{16} & \multicolumn{2}{|c|}{63.90} & \multicolumn{2}{|c|}{165.00} & \multicolumn{2}{|c|}{1.70} & \multicolumn{2}{|c|}{5338} \\
\hline & & 50 & 88 & 156 & 172 & 1.52 & 1.96 & 4303 & 6380 \\
\hline \multirow{2}{*}{$41-50$} & \multirow{2}{*}{10} & \multicolumn{2}{|c|}{60.30} & \multicolumn{2}{|c|}{166.00} & \multicolumn{2}{|c|}{1.67} & \multicolumn{2}{|c|}{5380} \\
\hline & & 54 & 71 & 159 & 171 & 1.54 & 1.79 & 4900 & 5380 \\
\hline \multirow{2}{*}{$51-60$} & \multirow{2}{*}{3} & \multicolumn{2}{|c|}{55.80} & \multicolumn{2}{|c|}{164.00} & \multicolumn{2}{|c|}{1.64} & \multicolumn{2}{|c|}{4981} \\
\hline & & 55 & 66 & 161 & 170 & 1.57 & 1.76 & 4387 & 5321 \\
\hline
\end{tabular}

Tabla 1. Valores promedio de edad, peso, talla, superficie corporal y capacidad vital en 56 hombres sanos, nativos, residentes del altiplano chileno (Isluga 3800-4100 m.snm).

\begin{tabular}{|c|c|c|c|c|c|c|c|c|c|}
\hline \multicolumn{10}{|c|}{ Mujeres } \\
\hline Edad & $\mathbf{N}^{\circ}$ & \multicolumn{2}{|c|}{ Peso (k) } & \multicolumn{2}{|c|}{ Talla (cm) } & \multicolumn{2}{|c|}{ Superficie $\left(\mathbf{m}^{2}\right)$} & \multicolumn{2}{|c|}{ Capacidad vital (ml) } \\
\hline \multirow{2}{*}{$15-20$} & \multirow{2}{*}{12} & \multicolumn{2}{|c|}{53.60} & \multicolumn{2}{|c|}{149.93} & \multicolumn{2}{|c|}{1.49} & \multicolumn{2}{|c|}{3960} \\
\hline & & 38 & 64 & 140 & 162 & 1.22 & 1.65 & 2926 & 4547 \\
\hline \multirow{2}{*}{$21-30$} & \multirow{2}{*}{17} & \multicolumn{2}{|c|}{54.20} & \multicolumn{2}{|c|}{150.83} & \multicolumn{2}{|c|}{1.49} & \multicolumn{2}{|c|}{3923} \\
\hline & & 47 & 68 & 145 & 155 & 1.37 & 1.67 & 2738 & 482 \\
\hline \multirow{2}{*}{$31-40$} & \multirow{2}{*}{7} & \multicolumn{2}{|c|}{59.50} & \multicolumn{2}{|c|}{151.29} & \multicolumn{2}{|c|}{1.55} & \multicolumn{2}{|c|}{3484} \\
\hline & & 52 & 78 & 146 & 157 & 1.42 & 1.78 & 3002 & 1931 \\
\hline \multirow{2}{*}{$41-50$} & \multirow{2}{*}{6} & \multicolumn{2}{|c|}{58.20} & \multicolumn{2}{|c|}{149.81} & \multicolumn{2}{|c|}{1.52} & \multicolumn{2}{|c|}{3401} \\
\hline & & 45 & 65 & 114 & 152 & 1.18 & 1.60 & 2743 & 1957 \\
\hline
\end{tabular}

Tabla 2. Valores de edad, peso, talla, superficie corporal y capacidad vital en 42 mujeres sanas, nativas, residentes del altiplano chileno (Isluga 3800-4100 m.snm).

\begin{tabular}{|c|c|c|c|c|c|c|c|c|c|}
\hline \multicolumn{10}{|c|}{ Hombres } \\
\hline Edad & $\mathbf{N}^{\mathbf{o}}$ & \multicolumn{2}{|c|}{ Peso (k) } & \multicolumn{2}{|c|}{ Talla (cm) } & \multicolumn{2}{|c|}{ Superficie $\left(\mathbf{m}^{2}\right)$} & \multicolumn{2}{|c|}{ Capacidad vital (ml) } \\
\hline \multirow{2}{*}{$21-30$} & \multirow{2}{*}{50} & \multicolumn{2}{|c|}{64.65} & \multicolumn{2}{|c|}{169.18} & \multicolumn{2}{|c|}{1.74} & \multicolumn{2}{|c|}{5355} \\
\hline & & 47.5 & 96.0 & 154 & 189 & 1.48 & 2.21 & 3748 & 6950 \\
\hline \multirow{2}{*}{$31-40$} & \multirow{2}{*}{50} & \multicolumn{2}{|c|}{71.70} & \multicolumn{2}{|c|}{169.10} & \multicolumn{2}{|c|}{1.82} & \multicolumn{2}{|c|}{5157} \\
\hline & & 53.7 & 105.5 & 156 & 181 & 1.58 & 2.23 & 3880 & 6769 \\
\hline \multirow{2}{*}{$41-50$} & \multirow{2}{*}{48} & \multicolumn{2}{|c|}{74.56} & \multicolumn{2}{|c|}{168.04} & \multicolumn{2}{|c|}{1.84} & \multicolumn{2}{|c|}{4679} \\
\hline & & 50.4 & 100.0 & 154 & 180 & 1.47 & 2.15 & 3500 & 6780 \\
\hline \multirow{2}{*}{$51-60$} & \multirow{2}{*}{27} & \multicolumn{2}{|c|}{73.91} & \multicolumn{2}{|c|}{167.96} & \multicolumn{2}{|c|}{1.83} & \multicolumn{2}{|c|}{4743} \\
\hline & & 54.0 & 92.1 & 1.56 & 185 & 1.53 & 2.10 & 3762 & 5628 \\
\hline \multirow{2}{*}{$\begin{array}{l}61 \text { y } \\
\text { más }\end{array}$} & \multirow{2}{*}{6} & \multicolumn{2}{|c|}{72.02} & \multicolumn{2}{|c|}{164.33} & \multicolumn{2}{|c|}{1.78} & \multicolumn{2}{|c|}{4548} \\
\hline & & 58.4 & $\mathrm{XxX} \cdot \mathrm{X}^{5}$ & 154 & 179 & 1.66 & 2.05 & 3324 & 5918 \\
\hline
\end{tabular}

Tabla 3. Valores promedio de edad, peso, talla, superficie corporal y capacidad vital en 181 hombres residentes de Chuquicamata (2800 m.snm).

5 Nota de la Editora (2009): x = Ininteligible en el original. 
CAPACIDAD VITAL, TALLA, PESO Y SUPERFICIE CORPORAL EN DOS POBLACIONES ADULTAS SANAS...

\begin{tabular}{|c|c|c|c|c|c|c|c|c|c|}
\hline Edad & $\mathbf{N}^{\mathbf{o}}$ & \multicolumn{2}{|c|}{ Peso (k) } & \multicolumn{2}{|c|}{ Talla (cm) } & \multicolumn{2}{|c|}{ Superficie $\left(\mathbf{m}^{2}\right)$} & \multicolumn{2}{|c|}{ Capacidad vital (ml) } \\
\hline \multirow{2}{*}{$18-20$} & \multirow{2}{*}{10} & \multirow{2}{*}{\multicolumn{2}{|c|}{52.40}} & \multicolumn{2}{|c|}{156.20} & \multicolumn{2}{|c|}{1.50} & \multicolumn{2}{|c|}{3780} \\
\hline & & & & 148 & 164 & 1.32 & 1.65 & 3366 & 4140 \\
\hline \multirow{2}{*}{$21-30$} & \multirow{2}{*}{50} & \multicolumn{2}{|c|}{55.40} & \multirow{2}{*}{\multicolumn{2}{|c|}{155.80}} & \multicolumn{2}{|c|}{1.54} & \multicolumn{2}{|c|}{3745} \\
\hline & & 40.0 & 74.0 & & & 1.30 & 1.76 & 2260 & 4575 \\
\hline \multirow{2}{*}{$31-40$} & \multirow{2}{*}{38} & \multicolumn{2}{|c|}{57.60} & \multicolumn{2}{|c|}{155.29} & \multicolumn{2}{|c|}{1.56} & \multicolumn{2}{|c|}{$371 x$} \\
\hline & & 42.5 & 74.0 & 143 & 167 & 1.29 & 1.76 & 2687 & 4644 \\
\hline \multirow{2}{*}{$41-50$} & \multirow{2}{*}{40} & \multirow{2}{*}{\multicolumn{2}{|c|}{65.70}} & \multicolumn{2}{|c|}{155.50} & \multicolumn{2}{|c|}{1.60} & \multicolumn{2}{|c|}{$\mathrm{xxxx}$} \\
\hline & & & & 141 & 163 & 1.32 & 1.90 & $247 x$ & 4516 \\
\hline \multirow{2}{*}{$51-60$} & \multirow{2}{*}{25} & \multicolumn{2}{|c|}{68.40} & \multicolumn{2}{|c|}{ XXX.XX } & \multicolumn{2}{|c|}{ X.Xx } & \multicolumn{2}{|c|}{$\mathrm{XXXX}$} \\
\hline & & 47.0 & 100.0 & 145 & 167 & 1.xx & 1.xx & $\mathrm{xxxx}$ & $\operatorname{xxxx}$ \\
\hline \multirow{2}{*}{$\begin{array}{l}61 \text { y } \\
\text { más }\end{array}$} & \multirow{2}{*}{$\mathrm{xx}$} & \multicolumn{2}{|c|}{$\mathrm{xx}$} & \multicolumn{2}{|c|}{$\mathrm{xx}$} & & & & \\
\hline & & $\mathrm{xX}$ & $\mathrm{xx}$ & $\mathrm{xX}$ & $\mathrm{XX}$ & $\mathrm{xx}$ & $\mathrm{xX}$ & $\mathrm{xx}$ & $x x^{6}$ \\
\hline
\end{tabular}

Tabla 4. Valores promedio de edad, peso, talla, superficie corporal y capacidad vital en 170 mujeres residentes de Chuquicamata (2800 m.snm).

\begin{tabular}{|c|c|c|c|c|c|c|c|c|c|}
\hline \multirow{2}{*}{ Edad } & \multicolumn{5}{|c|}{ Hombres } & \multicolumn{4}{|c|}{ Mujeres } \\
\hline & Loc. & $\mathbf{N}^{0}$ & Peso & Talla & Sup. & $\mathrm{K}$ ? & Peso & Talla & Sup. \\
\hline $15-20$ & $\begin{array}{l}\text { Isluga } \\
\text { Chuquicamata }\end{array}$ & $\begin{array}{r}13 \\
-\end{array}$ & $\begin{array}{r}55.90 \\
-\end{array}$ & $\begin{array}{r}163.00 \\
-\end{array}$ & $\begin{array}{r}1.59 \\
-\end{array}$ & $\begin{array}{l}12 \\
10\end{array}$ & $\begin{array}{l}53.39 \\
52.40\end{array}$ & $\begin{array}{l}149.83 \\
156.20\end{array}$ & $\begin{array}{l}1.49 \\
1.50\end{array}$ \\
\hline $21-30$ & $\begin{array}{l}\text { Isluga } \\
\text { Chuquicamata }\end{array}$ & $\begin{array}{l}14 \\
50\end{array}$ & $\begin{array}{l}58.60 \\
64.65\end{array}$ & $\begin{array}{l}165.00 \\
169.38\end{array}$ & $\begin{array}{l}1.64 \\
1.74\end{array}$ & $\begin{array}{l}17 \\
50\end{array}$ & $\begin{array}{l}54.20 \\
55.40\end{array}$ & $\begin{array}{l}150.88 \\
155.80\end{array}$ & $\begin{array}{l}1.49 \\
1.94\end{array}$ \\
\hline $31-40$ & $\begin{array}{l}\text { Isluga } \\
\text { Chuquicamata }\end{array}$ & $\begin{array}{l}16 \\
50\end{array}$ & $\begin{array}{l}63.90 \\
71.70\end{array}$ & $\begin{array}{l}165.00 \\
169.10\end{array}$ & $\begin{array}{l}1.70 \\
1.82\end{array}$ & $\begin{array}{r}7 \\
38\end{array}$ & $\begin{array}{l}59.50 \\
57.60\end{array}$ & $\begin{array}{l}151.29 \\
155.29\end{array}$ & $\begin{array}{l}1.55 \\
1.56\end{array}$ \\
\hline $41-50$ & $\begin{array}{l}\text { Isluga } \\
\text { Chuquicamata }\end{array}$ & $\begin{array}{l}10 \\
48\end{array}$ & $\begin{array}{l}60.30 \\
74.56\end{array}$ & $\begin{array}{l}166.00 \\
168.04\end{array}$ & $\begin{array}{l}1.67 \\
1.84\end{array}$ & $\begin{array}{r}6 \\
40\end{array}$ & $\begin{array}{l}58.20 \\
65.70\end{array}$ & $\begin{array}{l}149.83 \\
155.50\end{array}$ & $\begin{array}{l}1.52 \\
1.65\end{array}$ \\
\hline $51-60$ & $\begin{array}{l}\text { Isluga } \\
\text { Chuquicamata }\end{array}$ & $\begin{array}{r}3 \\
27\end{array}$ & $\begin{array}{l}58.80 \\
73.91\end{array}$ & $\begin{array}{l}164.00 \\
167.96\end{array}$ & $\begin{array}{l}1.64 \\
1.83\end{array}$ & - & 63.40 & 153.84 & 1.65 \\
\hline $\begin{array}{l}61 \text { y } \\
\text { más }\end{array}$ & $\begin{array}{l}\text { Isluga } \\
\text { Chuquicamata }\end{array}$ & $\overline{6}$ & 72.02 & 164.33 & 1.78 & $\overline{7}$ & $\begin{array}{r}- \\
67.90\end{array}$ & $\begin{array}{r}- \\
151.57\end{array}$ & 1.61 \\
\hline
\end{tabular}

Tabla 5. Peso, talla y superficie corporal en 56 hombres y 42 mujeres de Isluga y 181 hombres y 170 mujeres de Chuquicamata (todos sanos). Comparación según edad y sexo (promedios) (Isluga: 3800-4100 m.snm; Chuquicamata: 2800 m.snm).

\begin{tabular}{|c|c|c|c|c|c|c|c|}
\hline Edad & Loc. & $\mathbf{N}^{o}$ & Peso & Talla & Superficie & Capacidad vital & C.V. ${ }^{\text {Lts. mt }}$ \\
\hline $15-20$ & $\begin{array}{l}\text { Isluga } \\
\text { Chuquicamata }\end{array}$ & $\begin{array}{r}13 \\
-\end{array}$ & $\begin{array}{r}55.90 \\
-\end{array}$ & $\begin{array}{r}163.00 \\
-\end{array}$ & $\begin{array}{r}1.59 \\
-\end{array}$ & $\begin{array}{r}121.8 \\
-\end{array}$ & $\begin{array}{r}3.402 \\
-\end{array}$ \\
\hline $21-30$ & $\begin{array}{l}\text { Isluga } \\
\text { Chuquicamata }\end{array}$ & $\begin{array}{l}14 \\
50\end{array}$ & $\begin{array}{l}58.60 \\
64.65\end{array}$ & $\begin{array}{l}165.00 \\
169.38\end{array}$ & $\begin{array}{l}1.64 \\
1.64\end{array}$ & $\begin{array}{l}123.4 \\
113.0\end{array}$ & $\begin{array}{l}3.352 \\
3.000\end{array}$ \\
\hline $31-40$ & $\begin{array}{l}\text { Isluga } \\
\text { Chuquicamata }\end{array}$ & $\begin{array}{l}16 \\
50\end{array}$ & $\begin{array}{l}63.90 \\
71.70\end{array}$ & $\begin{array}{l}165.00 \\
169.10\end{array}$ & $\begin{array}{l}1.70 \\
1.82\end{array}$ & $\begin{array}{l}125.0 \\
115.5\end{array}$ & $\begin{array}{l}3.171 \\
2.829\end{array}$ \\
\hline $41-50$ & $\begin{array}{l}\text { Isluga } \\
\text { Chuquicamata }\end{array}$ & $\begin{array}{l}10 \\
48\end{array}$ & $\begin{array}{l}60.30 \\
74.56\end{array}$ & $\begin{array}{l}166.00 \\
168.04\end{array}$ & $\begin{array}{l}1.67 \\
1.84\end{array}$ & $\begin{array}{l}132.1 \\
112.2\end{array}$ & $\begin{array}{l}3.230 \\
2.537\end{array}$ \\
\hline $51-60$ & $\begin{array}{l}\text { Isluga } \\
\text { Chuquicamata }\end{array}$ & $\begin{array}{r}3 \\
27\end{array}$ & $\begin{array}{l}58.80 \\
73.01\end{array}$ & $\begin{array}{l}164.00 \\
167.96\end{array}$ & $\begin{array}{l}1.64 \\
1.83\end{array}$ & $\begin{array}{l}131.2 \\
117.2\end{array}$ & $\begin{array}{l}3.000 \\
2.55 \mathrm{x}\end{array}$ \\
\hline $\begin{array}{l}61 \text { y } \\
\text { más }\end{array}$ & $\begin{array}{l}\text { Isluga } \\
\text { Chuquicamata }\end{array}$ & $\frac{-}{6}$ & $72.0 \overline{2}$ & 164.33 & $\begin{array}{r}- \\
1.78\end{array}$ & $131 . \overline{6}$ & 2.580 \\
\hline
\end{tabular}

Tabla 6. Valores promedio de peso, talla, superficie corporal y capacidad vital (\% y Lts. BIPS/Mt $\left.{ }^{2}\right)$ en 56 hombres de Isluga y 181 hombres de Chuquicamata. Comparación según grupos de edad (Isluga: 3800-4100 m.snm; Chuquicamata: 2800 m.snm).

6 Nota de la Editora: $\mathrm{x}=$ Ininteligible en el original. 


\begin{tabular}{|c|c|c|c|c|c|c|c|}
\hline Edad & Loc. & $\mathbf{N}^{o}$ & Peso & Talla & Superficie & Capacidad vital & C.V..$^{\text {Lts. mt }}$ \\
\hline $15-20$ & $\begin{array}{l}\text { Isluga } \\
\text { Chuquicamata }\end{array}$ & $\begin{array}{l}12 \\
10\end{array}$ & $\begin{array}{l}53.30 \\
52.40\end{array}$ & $\begin{array}{l}149.83 \\
156.20\end{array}$ & $\begin{array}{r}1.49 \\
150\end{array}$ & $\begin{array}{l}125.4 \\
111.2\end{array}$ & $\begin{array}{l}2.670 \\
2.496\end{array}$ \\
\hline $21-30$ & $\begin{array}{l}\text { Isluga } \\
\text { Chuquicamata }\end{array}$ & $\begin{array}{l}17 \\
50\end{array}$ & $\begin{array}{l}54.20 \\
55.40\end{array}$ & $\begin{array}{l}150.88 \\
155.80\end{array}$ & $\begin{array}{l}1.49 \\
1.54\end{array}$ & $\begin{array}{l}128.7 \\
115.1\end{array}$ & $\begin{array}{r}2.645 \\
410\end{array}$ \\
\hline $31-40$ & $\begin{array}{l}\text { Isluga } \\
\text { Chuquicamata }\end{array}$ & $\begin{array}{r}7 \\
38\end{array}$ & $\begin{array}{r}5950 \\
57.60\end{array}$ & $\begin{array}{l}151.20 \\
155.29\end{array}$ & $\begin{array}{l}1.55 \\
1.56\end{array}$ & $\begin{array}{l}120 . x \\
121.0\end{array}$ & $\begin{array}{r}\mathrm{xxxx} \\
2.390\end{array}$ \\
\hline $41-50$ & $\begin{array}{l}\text { Isluga } \\
\text { Chuquicamata }\end{array}$ & $\begin{array}{r}6 \\
40\end{array}$ & $\begin{array}{l}58.20 \\
65.70\end{array}$ & $\begin{array}{l}149.83 \\
155.50\end{array}$ & $\begin{array}{l}1.52 \\
1.65\end{array}$ & $\begin{array}{l}\text { 110.x } \\
\text { 119.x }\end{array}$ & $\begin{array}{l}2.23 x \\
2.123\end{array}$ \\
\hline $51-60$ & $\begin{array}{l}\text { Isluga } \\
\text { Chuquicamata }\end{array}$ & - & 68.4 & 153.84 & 1.65 & 121.6 & $\begin{array}{r}- \\
1.940\end{array}$ \\
\hline $\begin{array}{l}61 \text { y } \\
\text { más }\end{array}$ & $\begin{array}{l}\text { Isluga } \\
\text { Chuquicamata }\end{array}$ & $\overline{7}$ & 67.90 & $\begin{array}{r}- \\
151.57\end{array}$ & $\begin{array}{r}- \\
1.64\end{array}$ & $111 . \overline{6}$ & 1.575 \\
\hline
\end{tabular}

Tabla 7. Valores promedio de peso, talla, superficie corporal y capacidad vital (\% y lt BIPS/Mt ${ }^{2}$ ) en 42 mujeres de Isluga y 170 mujeres de Chuquicamata. Comparación según grupos de edad (Isluga: 3800-4100 m.snm; Chuquicamata: 2800 m.snm).

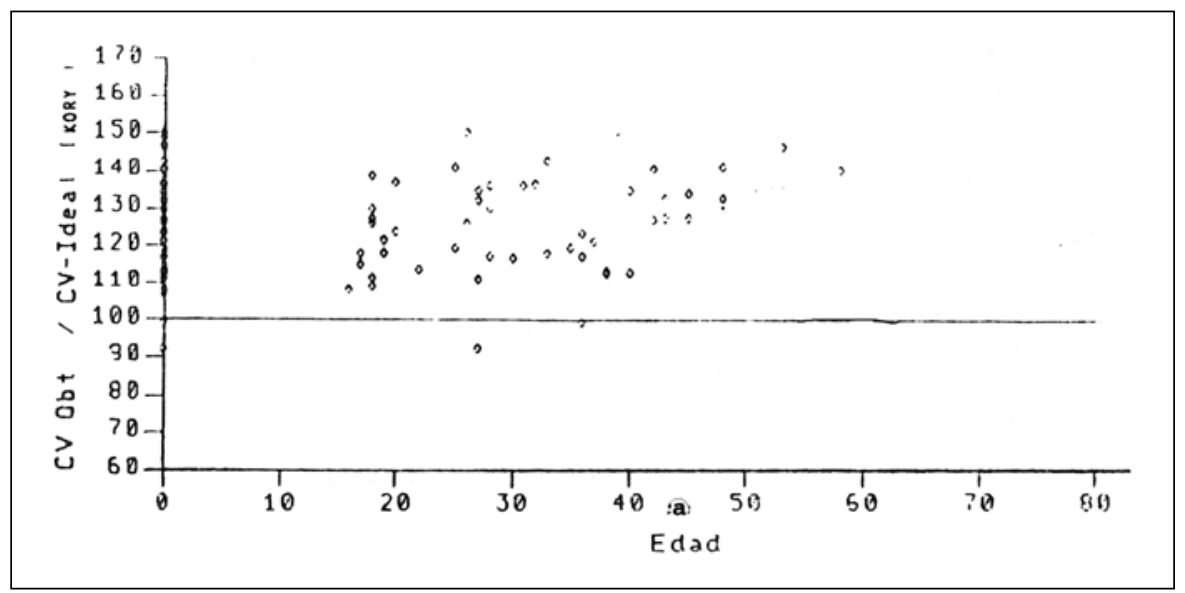

Gráfico 1. CV Obtenida/CV-Ideal (Kory) * 100 en 56 hombres sanos nativos residentes del altiplano chileno. (Bofedal Isluga 3800 a 4100 m.snm).

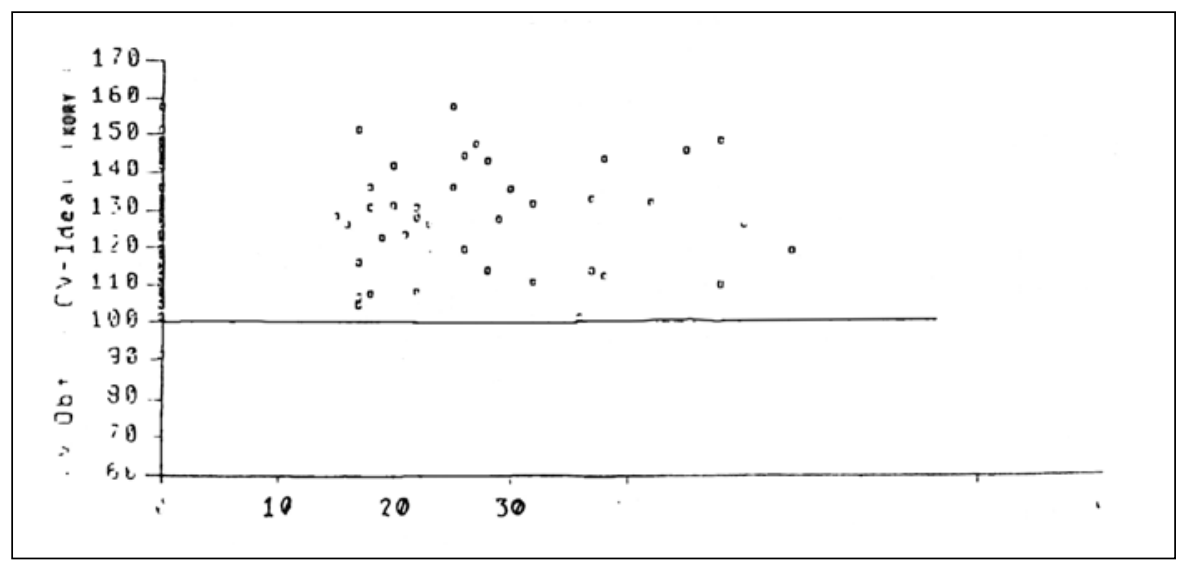

Gráfico 2. CV Obtenida/CV-Ideal (Kory) * 100 en 42 mujeres sanas nativas residentes del altiplano chileno. (Bofedal Isluga 3800 a 4100 m.snm). 


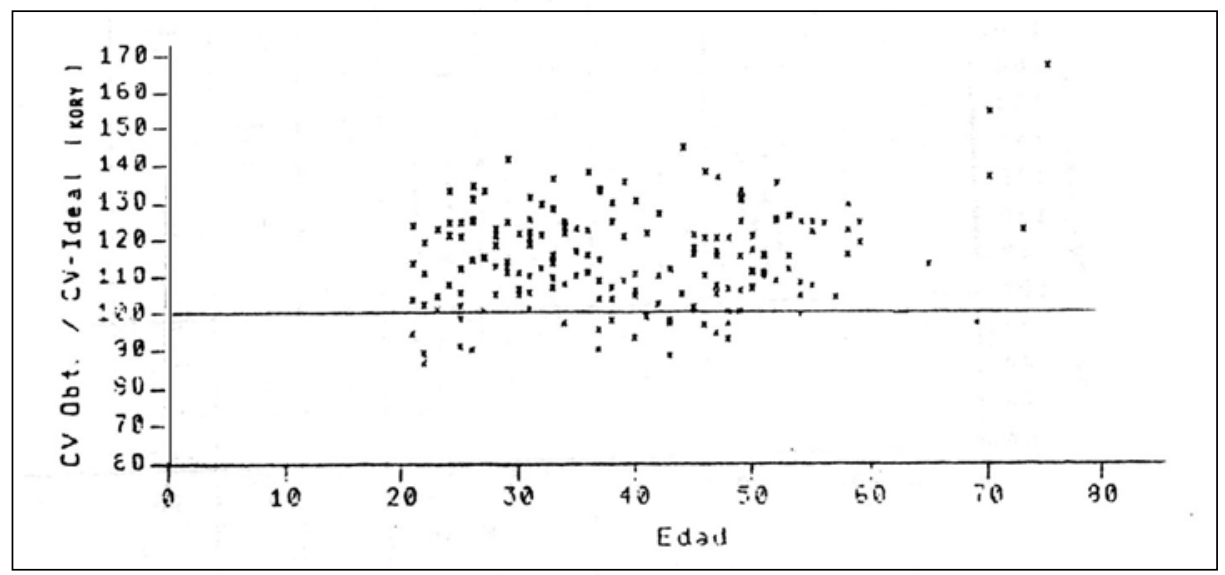

Gráfico 3. CV Obtenida/CV-Ideal (Kory) * 100 en 181 hombres sanos trabajadores no mineros residentes en Chuquicamata (2800 m.snm).

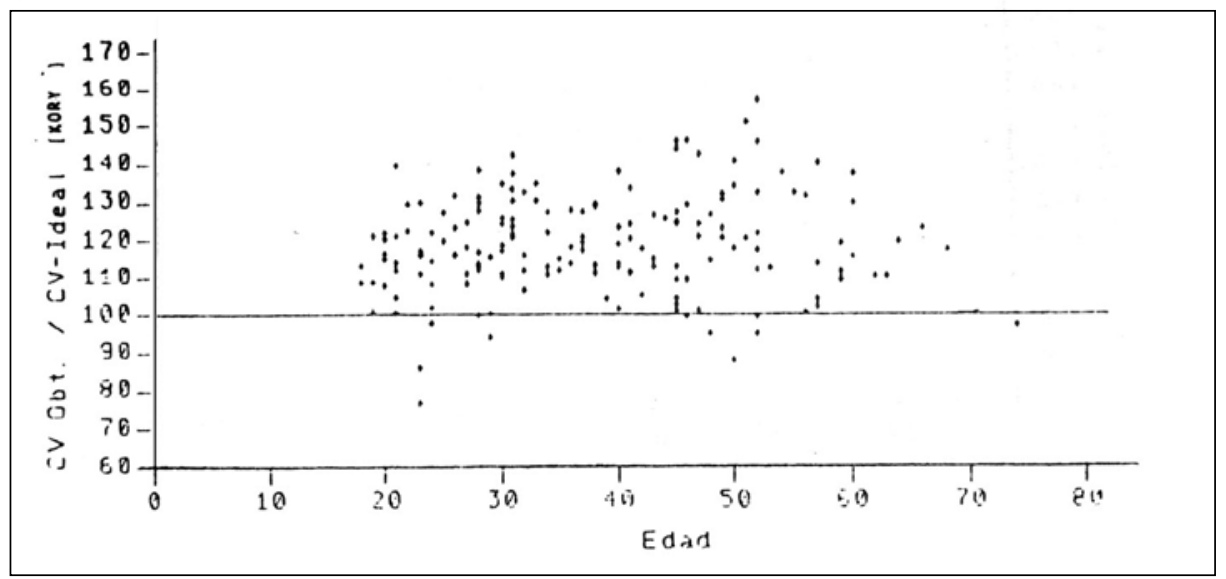

Gráfico 4. CV Obtenida/CV-Ideal (Kory) * 100 en 170 mujeres sanas trabajadoras no mineras residentes en Chuquicamata (2800 m.snm).

a la diferencia de etnias, alimentación, grado de aclimatación, etc., que separa a ambos grupos. La homogeneidad del grupo de Isluga se demuestra también en la escasa dispersión de los valores extremos de estatura, en comparación con lo que ocurre en Chuquicamata.

La diferencia de talla entre los indígenas de Isluga y los publicados por otros autores para indígenas peruanos de Nuñoa, Huancayo y bolivianos, de los alrededores del Lago Titicaca, puede explicarse por muchos factores diferenciales. No hemos practicado en la población estudiada encuestas alimenticias que pudieran añadir elementos sobre este tema. Sin embargo, el hecho de corresponder nuestro grupo a una comunidad geográfica y culturalmente más cerrada, con persistencia de patrones culturales y, quizás, genéticos más puros, puede constituir una vertiente de explicación. Todos ellos hablaban aymara y algunos, aun el puquina. Su vestimenta, particularmente femenina, difería notoriamente de la habitual en el altiplano boliviano vecino, lo que unido a otras consideraciones permite sospechar cierta raíz uro-chipaya en el grupo. Los habitantes altiplánicos bolivianos que habitan en alturas vecinas semejantes poseen tallas promedio de $160.3 \mathrm{~cm}$ inferiores a la de nuestro grupo pero mayores que los $158.8 \mathrm{~cm}$ de los nativos andinos peruanos. Sólo conocemos el estudio de 20 láguidos, de origen uro chipaya estudiados, cuya talla de $159.1 \mathrm{~cm}$ es menor que la referida por nosotros. No hemos medido otros índices morfológicos, como los de cráneo y cara, que pudieran añadir elementos de interés en el problema (Vellard 1970). 
En los distintos grupos de edad en ambos sexos los nativos de Isluga no mostraron gran dispersión en lo referente a los valores promedio y peso corporal (Tablas 1 y 2). La Tabla 5 compara este grupo con el de Chuquicamata. Allí se aprecia que la diferencia de peso entre el grupo más joven y el más viejo entre los hombres de Chuquicamata varió en 9.26 k y en las mujeres en $15.5 \mathrm{k}$. En Isluga esta diferencia fue sólo de 2.9 y 4.9 k respectivamente. La dispersión en cada grupo etario y en ambos sexos respecto del valor promedio de peso corporal es mucho mayor en Chuquicamata (Tablas 3 y 4), grupo cuya prevalencia de obesidad es alta a diferencia de lo que ocurre en Isluga.

Los valores de superficie corporal (Tabla 5) demuestran superficies mucho mayores en hombres y mujeres de todas las edades entre los residentes de Chuquicamata respecto de los nativos de Isluga.

En las Tablas 1 a 4 se anotan también los valores promedio y el rango de la capacidad vital, distribuidos por grupos etarios. La capacidad vital es el máximo volumen de gas que puede ser expulsado de los pulmones después de una inspiración máxima. Se trata de una prueba simple, fácilmente reproducible, que se utiliza rutinariamente como prueba cuantitativa de capacidad y elasticidad del continente toracopulmonar. Su disminución es sinónimo de alteración restrictiva, primaria o secundaria del pulmón. Representa el mayor porcentaje del aire existente en el pulmón al finalizar una inspiración máxima, valor este conocido como capacidad pulmonar total. La espiración máxima desde ese nivel mantendrá siempre un volumen de aire dentro del pulmón: el volumen residual, que sólo puede medirse por métodos indirectos. La relación entre volumen residual y capacidad pulmonar total es de $20 \%$ en el joven sano pero alcanza a $35 \%$ en el viejo sano. Esta hiperinflación del pulmón del viejo, en ausencia de enfermedad obstructiva, se correlaciona con la tendencia inversa de la capacidad vital que disminuye a medida que la edad avanza (Comroe et al. 1956; Boren et al. 1966; West 1975; Dejours 1982).

La capacidad vital es mayor en los hombres que en las mujeres; disminuye con la edad y, por tratarse de un valor estrictamente individual, se han buscado tablas de valores normales que permitan en cada caso conocer el volumen teórico, supuesto ideal. Las tablas más usadas correlacionan la capacidad vital, para cada sexo y edad, con la estatura, factor que posee la mejor correlación. Las tablas y nomogramas usados con alto margen de seguridad en la población norteamericana se aplican a nuestra realidad indoiberoamericana, sin estudios previos para evaluar su vigencia. Desconocemos tablas orientales que quizá pudieran ser más adecuadas a nuestra realidad, debido a la ausencia de estudios nacionales o sudamericanos de alternativa.

El hombre andino posee una mayor circunferencia torácica y menor estatura que el norteamericano de igual edad y la altura de su esternón y el diámetro anteroposterior del tórax son mayores que los habitantes de las tierras bajas de nuestros países (Monge et al. 1954). La circunferencia torácica posee un coeficiente de correlación con capacidad vital muy alta en los residentes de altura (0.9), comparado con los del nivel del mar (0.28). Esta correlación es mucho mayor en la altura que con la estatura (0.60). La correlación en residentes de la costa de Perú entre capacidad vital y talla es de 0.70 (Frisancho 1960).

En las Tablas 6 y 7 se aprecia que el promedio de los valores de capacidad vital de los individuos estudiados en Isluga y en Chuquicamata se encuentra francamente por encima del supuesto ideal obtenido de la tabla de Kory, norma norteamericana usada en Chile. Este promedio fluctúa para cada grupo de edad entre 121 y $130 \%$, alcanzando en casos individuales valores superiores al $150 \%$ en Isluga, fluctuando entre 122 y $121 \%$ en el grupo Chuquicamata.

En los Gráficos 1 a 4 se expresan todos los valores individuales como porcentaje de la tabla de Kory. Se aprecia que prácticamente todos los valores se encuentran muy por encima de la norma norteamericana.

Por tratarse de un trabajo preliminar no hemos calculado coeficiente de correlación ni líneas de regresión, tampoco hemos descartado especialmente en Chuquicamata algunos casos que mostraron al examen físico o radiológico de tórax signos compatibles con alguna patología discreta capaz de restringir el parámetro estudiado. Finalmente hemos incluido todos los valores, aunque muchos de ellos en especial en Chuquicamata mostraban en las demás pruebas espirométricas signos discretos o moderados de aumento de resistencia al flujo aéreo en las vías.

Las diferencias entre el grupo de Isluga y Chuquicamata son significativas en el sentido de que en promedio para igual talla, edad y sexo, la capacidad vital es mayor entre los primeros. En las Tablas 6 y 7 ello es evidente expresando la capacidad vital en litros BTPS por $\mathrm{m}^{2}$ de superficie corporal. Esta diferencia 
sería aún mayor si se efectuara la correlación relativa a la obesidad.

Las diferencias entre los nativos de Isluga y los residentes de Chuquicamata pueden explicarse por múltiples factores:

1. Nivel de altura. Esta correlación entre capacidad vital, circunferencia torácica, volumen torácico, diámetro anteroposterior del tórax y altura del esternón con el nivel de altura de la residencia ya ha sido certificada por otros autores (Monge et al. 1954).

2. Razas. El grupo de Isluga representa una comunidad étnica prácticamente homogénea, lo que no ocurre en el grupo de Chuquicamata en el que existe un fuerte componente caucasoide.

3. Grado de aclimatación a la altura. La comunidad de Isluga estudiada es como toda ella nativa de las grandes alturas residente permanente en ella. La excelencia de su aclimatación puede certificarse por los altos valores de capacidad aeróbica que poseen hasta edades avanzadas (Donoso y Santolaya 1971; Santolaya et al. 1973). La de Chuquicamata es muy heterogénea y su tiempo variable de residencia en la mediana altura será estudiado al finalizar el trabajo, mediante computación.

4. Hábitos de vida. El grupo de Isluga muestra escasa prevalencia de obesidad, ausencia de consumo de tabaco y alto nivel de actividad física, situación no comparable a la vigente en Chuquicamata donde la obesidad, el sedentarismo y el hábito de fumar son frecuentes.

\section{Resumen}

1. Se presentan los resultados de talla, peso, superficie corporal y capacidad vital estudiadas en dos poblaciones adultas, de ambos sexos residentes de altura en el Norte Grande de Chile: 98 nativos del altiplano chileno, del bofedal de Isluga $(3800 \mathrm{~m})$ y 351 residentes permanentes sanos trabajadores no mineros del campamento cuprífero de Chuquicamata (2800 m).

2. Se discuten las diferencias entre ambos grupos, particularmente las relativas a raza; calidad de la aclimatación a la altura; nivel de altitud; hábitos de vida (alimentación, obesidad, ejercicio, tabaco).

3. Se demuestra que la talla, el peso y la superficie corporal del grupo de Chuquicamata es mayor que la del grupo Isluga en ambos sexos y para todas las edades.

4. Se demuestra que la capacidad absoluta y relativa a la talla superficie es mayor en el grupo Isluga que Chuquicamata, corroborando otras publicaciones. Los valores de capacidad vital, comparados con la tabla de Kory norma norteamericana de uso habitual en nuestro medio, demuestran cifras superiores a las supuestas ideales para el sexo, edad y talla. Este comportamiento ocurre en ambos grupos pero es más relevante en el grupo Isluga.

5. Se concluye, ratificando publicaciones internacionales preferentemente peruanas que la correlación de capacidad vital y talla en las poblaciones andinas es poco significativa, lo que hace dudosa la aplicabilidad de las normas norteamericanas habitualmente usadas.

\section{REFERENCIAS CITADAS}

BOREN, H. G., R. C. KORY. y J. C. SYNER, 1966. Prediction normogram for normal men. American Journal of Medical Science 41: 96-114.

BURRI, P. H. y E. R. WEIBEL, 1970. Experientia 26: 68.

- 1971. Morphometric evaluation of changes in lung structure due to high altitude. High altitude physiology cardiac and respiratory aspects. Ciba Foundation Symposium (Churchill Livingstone). Edimburgo y Londres.

CARDICH, A., 1964. Lauricocha. Fundamentos para una prehistoria de los Andes Centrales. Centro Argentino de Estudios Prehistóricos, Buenos Aires.
— 1960. Investigación prehistórica de los Andes peruanos. Antiguo Perú. Espacio y tiempo. Semana de Arqueología Peruana (9-14 nov. de 1954). Editorial Mejía Baca, Lima.

CHEEK, D. B., I. E. GRAYSTONE y R. D. ROWE, 1969. American Journal of Physiology 217: 642-645.

COMROE, FORSTER, DUBOIS, BRISCOE y CARLSEN, 1956. The lung. The Year Book Medical Publication Inc., Chicago.

COON, C. S., S. M. GRAN y J. E. BIRSDELL, 1950. Races, a study of the problems of race formation in man. A Monography in American Lectures in Physical Anthropology Ill. Charles C. Thomas, Springfield. 
DEJOURS, P., 1982. Physiologie de la respiration. Flammarion Medical Sciences.

DONOSO, H., E. APUD, M. C. SAÑUDO y R. SANTOLAYA, 1971. Capacidad aeróbica como índice de adecuidad física en nuestras poblaciones (urbanas y nativas de altura) y en un grupo de atletas de selección. Revista Médica de Chile 99: 719-731.

DONOSO, H., R. SANTOLAYA y E. APUD, 1976. Hematocrit in native populations living permanently at high altitude in the Andean plateau of Northern Chile. Les Colloques de l'Institut de la Santé et de la Recherche Medicale 63: 547-552.

D'ORBIGNY, A., 1944. El hombre americano. Editorial Futuro, Buenos Aires.

FRISANCHO, R. A., 1960. Human growth and pulmonary function of a high altitude peruvian quechua population. Human Biology 41: 365.

1970. Developmental responses to high altitude hypoxia American Journal of Physical Anthropology 32: 401.

1977. Developmental adaptation to high altitude hipoxia. Int. Biometeor. 21: 135.

1978Ms. Crecimiento y desarrollo en las alturas: Síntesis integral a escala mundial. Curso internacional sobre antropología física y fisiología del habitante andino. Lima.

FRISANCHO, R. A. y P. T. BAKER, 1970. Altitude and growth a study of the patterns of physical growth of a high altitude peruvian quechua population. American Journal of Physical Anthropology 32: 279.

HOPKINS, D. M., 1962. Current Anthropology, junio.

HURTADO, A., 1964. Animals in high altitude: Resident man Handbook of Physiology: Environment cap. 54, pp. 843860.

KAUFFMANN-DOIG, F., 1978. Manual de arqueología peruana. Editorial Iberia. Lima.

KORY, R. C., H. G. CALLAHAN, J. BOREN y C. SYNER, 1961. Clinical spirometry in normal men. The veterans administration army cooperative study of pulmonary function I. M. Journal Med. 30: 243-258.
MONGE, C. y C. MONGE, 1967. Adaptación biológica de las sociedades americanas a las grandes alturas. Perú Indígena 26.

MONGE, C. y otros, 1954. Aclimatación en los Andes: Forma y función del tórax. Perú Indígena 5 (13): 3-15.

MOSTNY, G., 1976. Prehistoria de Chile. Editorial Universitaria, Santiago.

PANYELlA, A., 1975. Razas humanas. Editorial Sopena, Barcelona.

SANTOLAYA, R., 1981. Adaptación y aclimatación a la altura: Un proceso integral. Revista Médica del Hospital Roy $H$. Glover. 1: 51-60.

-1983Ms. Aclimatación a la altura. Simposio de Arqueología Atacameña, San Pedro de Atacama.

SANTOLAYA, R. y H. DONOSO, 1976. Estudio de la prevalencia de infección TBC, según la técnica de la tuberculina standard RT 23 con Tween 80 de 2 UT, intradérmica, en poblaciones nativas residentes de la precordillera y altiplano chileno. Les Colloques de l'Institut National de la Santé et de la Recherche Medicale 63.

SANTOLAYA, R., H. DONOSO, E. APUD y M. C. SAÑUDO, 1973. Electrocardiograma y capacidad aeróbica en nativos residentes de altura del altiplano chileno, como índice de aclimatación. Revista Médica de Chile 101: 433.

SANTOLAYA, R. y otros, 1981. Hematocrito, hemoglobina y presión de oxígeno arterial en 270 hombres y 266 mujeres sanas residentes de altura $(2800 \mathrm{~m})$. Revista Médica del Hospital Roy H. Glover 1: 17.

1981. Glicemia venenosa en ayunas en 532 residentes sanos de Chuquicamata (2800 m.snm). Revista Médica del Hospital Roy H. Glover 1 (2): 31.

TIMIRAS, P. S., A. A. KRUM y N. PACE, 1957. American Journal Physiology 191: 598-604.

VELLARD, J., 1970. Biotipo del habitante de la meseta andina. Anuario del Instituto Boliviano de Biología de Altura, pp. 7-30. La Paz.

WEST, J. B., 1975. Respiratory physiology. The essentials. The Williams and Wilkins Co., Baltimore. 